\title{
L'accumulo di alluminio nei pazienti uremici
}

\author{
A. Masti, M. Di Paolo, I. Barni Comparini, F. Centini
}

Dipartimento di Scienze Medico-Legali e Socio-Sanitarie - Università di Siena

N

ell'ultimo decennio la letteratura internazionale ha collegato alcune manifestazioni tossiche con l'assunzione di alluminio contenuto in numerosi farmaci somministrati talvolta in quantità elevate e per lunghi periodi di tempo nonché con l'utilizzazione in grandi quantità di soluzioni da infusione per uso medico contaminate con questo metallo (1-3). È stato infatti ipotizzato e dimostrato il coinvolgimento di tale elemento in molteplici patologie, quali, ad esempio, la demenza e la osteodistrofia renale che si manifestano nei soggetti sottoposti ad emodialisi (4-7) e la malattia di Alzheimer (8-10). Inoltre l'alluminio, in concentrazioni elevate, sembra interferire sul normale metabolismo dei globuli rossi (11-13), sulla funzione paratiroidea (14) e sui cromosomi (15).

La cinetica ed il metabolismo dell'alluminio sono stati recentemente oggetto di numerose ricerche al fine di individuarne con esattezza le vie di introduzione ed eliminazione, i limiti di accumulo nei vari organi, gli effetti tossici, nonché le possibilità di chelare il metallo una volta accumulato (16-18). Diversi studi hanno ricercato dati precisi sull'esposizione della popolazione attraverso gli alimenti giornalmente consumati nel convincimento che la dieta possa rappresentare il mezzo più rilevante di contatto ambientale per l'uomo sano e non professionalmente esposto (19).

Rimangono tuttavia aperti molti interrogativi: prima di tutto non è stato ancora chiarito, per la scarsità di dati presenti in letteratura, quale sia la quantità di allu- minio che si deve accumulare in un tessuto organico per provocare una malattia; a nostro parere, questa mancanza di precisione crea una serie di altri problemi connessi alla ottimizzazione della prevenzione, alla necessità di individuare mezzi diagnostici sempre più sensibili ed alla standardizzazione di protocolli terapeutici efficaci e sicuri.

Una revisione della letteratura ci ha permesso di confrontare i dati di vari Autori inerenti la presenza di alluminio in molti organi, tessuti e liquidi biologici di soggetti normali ed uremici (Tabb. I-II), evidenziando una disparità di risultati che non permettono ancora oggi, purtroppo, di dare un'indicazione precisa sui limiti dei valori normali.

Lo scopo della presente ricerca è stato quello di verificare i livelli di alluminio nel siero e nelle urine di un gruppo di pazienti affetti da insufficienza renale cronica e sottoposti a trattamento dialitico comparandoli con quelli di soggetti normali ed inoltre determinare le concentrazioni di alluminio nei tessuti di soggetti emodializzati deceduti e sottoposti a riscontro diagnostico comparandoli con un gruppo di controllo formato da soggetti deceduti per patologie diverse dall'uremia.

\section{Materiali e metodi}

Lo studio, condotto per tre anni presso il Policlinico dell'Università di Siena, ha considerato i livelli di alluminio nei seguenti materiali:
1) 111 campioni di siero provenienti da pazienti in trattamento emodialitico, di età media $60.8 \pm 13.1$ anni, età media dialitica (uomini e donne); e 45 campioni di siero provenienti da soggetti normali di

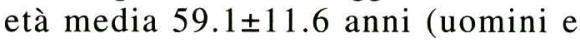
donne);

2) 47 campioni di urina provenienti dagli stessi pazienti in emodialisi con una diuresi conservata e 45 campioni di urina degli stessi soggetti di controllo;

3) la determinazione dell'alluminio nei tessuti è stata effettuata impiegando reperti autoptici prelevati da 12 soggetti dializzati deceduti e sottoposti a riscontro diagnostico ( 7 uomini e 5 donne, età media $70.25 \pm 11.09$ anni) e 10 soggetti non uremici sottoposti ad autopsia (2 donne e 8 uomini, età media 56.4+23.93 anni).

Per la determinazione dell'alluminio è stato utilizzato uno spettrometro per assorbimento atomico Varian mod. Spectra AA300 Zeeman, dotato di autocampionatore, equipaggiato con fornetto di grafite pirolitica "Partition Tube" (Varian) e gestito da un software IBM PS/2 mod. 30.

Il prelievo ematico è stato effettuato direttamente presso l'Unità Dialitica secondo la seguente procedura: con una siringa sono stati prelevati $10 \mathrm{ml}$ di sangue e trasferiti in una provetta contenente eparina sodica. Dopo agitazione e centrifugazione a $3000 \mathrm{rpm}$ per 10 minuti è stato prelevato $1 \mathrm{ml}$ di siero che ha costituito il campione da analizzare.

I campioni urinari, raccolti in provette di polypropylene sterile Falcon (Becton Dickinson \& C.), sono stati conservati alla temperatura di $2^{\circ} \mathrm{C}$. 
TABELLA I - CONCENTRAZIONI DI ALLUMINIO RISCONTRATE IN SOGGETTI EMODIALIZZATI

\begin{tabular}{|c|c|c|c|c|}
\hline AUTORE & $\begin{array}{c}\text { Siero o Plasma } \\
\mu \mathrm{g} / \mathrm{L}\end{array}$ & $\begin{array}{l}\text { Osso } \\
\mathrm{mg} / \mathrm{kg}\end{array}$ & $\begin{array}{l}\text { Fegato } \\
\mathrm{mg} / \mathrm{kg}\end{array}$ & $\begin{array}{c}\text { Cervello } \\
\mathrm{mg} / \mathrm{kg}\end{array}$ \\
\hline Minoia (20) & ----- & $14.9 \pm 3.7$ & ----- & --.- \\
\hline Andersen (21) & ---- & ----- & $30-300$ & $\begin{array}{l}24.8 \pm 3.3 \\
45.6 \pm 2.1\end{array}$ \\
\hline Minoia (22) & ----- & $6.51-99.76$ & ---- & ---- \\
\hline Valentin (23) & $6-254$ & ----- & ---- & $-\cdots$ \\
\hline Ward (6) & $97-242$ & ---- & ---- & ----- \\
\hline Alderman (24) & $50-600$ & ---- & ---- & ----- \\
\hline Gilli (25) & $31.07 \pm 24.6$ & --.- & ----- & ---- \\
\hline Gorsky (26) & $27-254.21$ & ---- & ----- & ---- \\
\hline Oster (27) & $8-713$ & $-\cdots$ & ----- & $-\cdots--$ \\
\hline Salvadeo (28) & $61.4 \pm 25.3$ & ---- & ---- & ---- \\
\hline Mattiello (29) & $12.1-237.8$ & $-\cdots$ & ---- & ----- \\
\hline \multirow[t]{3}{*}{ Sanna (30) } & $64 \% \pm 50$ & & & \\
\hline & $50<30 \% \leq 100$ & & & \\
\hline & $6 \%>100$ & ---- & --- & $-\cdots$ \\
\hline Cecco (31) & $0-210$ & $-\cdots$ & ---- & ----- \\
\hline Aucella (32) & $4-96$ & ---- & ---- & ----- \\
\hline Copercini (33) & $1-170$ & ---- & ---- & ---- \\
\hline
\end{tabular}

I reperti autoptici, prelevati al tavolo settorio usando forbici e pinze in acciaio, preventivamente lavate con soluzioni di acido nitrico $0.1 \%$ (Suprapure, Merk) ed EDTA (Ultrapure Reagent, IBI) e risciacquati con acqua distillata, sono stati conservati in congelatore ad una temperatura di $-10^{\circ} \mathrm{C}$. Per la loro analisi frammenti di circa $500 \mathrm{mg}$ prelevati da ciascun organo e tessuto sono stati posti in stufa a $110^{\circ} \mathrm{C}$, in un crogiolo in quarzo a fondo tondo della capacità di $5 \mathrm{~mL}$, preventivamente tarato, fino ad ottenere un peso secco costante. Dopo incenerimento in muffola alla temperatura di $550^{\circ} \mathrm{C}$ per circa 24 ore, i residui sono stati ripresi con $2 \mathrm{~mL}$ di acqua bidistillata, addizionati di $100 \mu \mathrm{l}$ di acido nitrico, lasciati sciogliere a freddo, trasferiti in una provetta e portati a $5 \mathrm{~mL}$ con acqua distillata. Per il sangue e per la bile l'indagine è stata condotta prelevando $1 \mathrm{~mL}$ di ciascuno dei due liquidi e diluendo fino ad ottenere un volume di $5 \mathrm{~mL}$ con acqua distillata. $100 \mu \mathrm{l}$ di siero, di urina e di soluzione di tessuto sono stati addizionati a $400 \mu \mathrm{ldi}$ soluzione di modificatore di matrice (2.5 g/L di $\mathrm{Mg}\left(\mathrm{NO}_{3}\right)$ Suprapure

TABELLA II - CONCENTRAZIONI DI ALLUMINIO IN SOGGETTI NORMALI

\begin{tabular}{|c|c|c|c|c|c|c|c|c|c|}
\hline AUTORE & $\begin{array}{l}\text { Siero } \\
\mu \mathrm{g} / \mathrm{L}\end{array}$ & Urina & $\begin{array}{l}\text { Milza } \\
\mathrm{mg} / \mathrm{kg}\end{array}$ & $\begin{array}{l}\text { Fegato } \\
\mathrm{mg} / \mathrm{kg}\end{array}$ & $\begin{array}{c}\text { Muscolo } \\
\mathrm{mg} / \mathrm{kg}\end{array}$ & $\begin{array}{l}\text { Cuore } \\
\mathrm{mg} / \mathrm{kg}\end{array}$ & $\begin{array}{l}\text { Polmone } \\
\mathrm{mg} / \mathrm{kg}\end{array}$ & $\begin{array}{c}\text { Cervello } \\
\mathrm{mg} / \mathrm{kg}\end{array}$ & $\begin{array}{l}\text { Osso } \\
\mathrm{mg} / \mathrm{kg}\end{array}$ \\
\hline Clavel (34) & $10-45$ & ----- & ---- & ---- & ---- & ---- & ---- & ---- & ---- \\
\hline Allain (35) & $12.5 \pm 4$ & $4.7 \pm 2.5 \mu \mathrm{g} / \mathrm{L}$ & ---- & ----- & ---- & ---- & ---- & --- & ---- \\
\hline Alderman (24) & $0.0 \pm 7.4$ & $0-7.6 \mu \mathrm{g} / \mathrm{L}$ & ----- & ---- & ---- & ---- & ---- & --- & ---- \\
\hline Oster (27) & $2.5-7$ & ----- & ---- & ---- & ---- & ---- & ---- & ---- & ---- \\
\hline Leung (36) & $2-14$ & ---- & ---- & ---- & ---- & ---- & ---- & ---- & ---- \\
\hline Parkinson (37) & $2-15$ & $2-15 \mu \mathrm{g} / \mathrm{L}$ & ----- & ---- & ---- & ---- & --- & $\cdots$ & ---- \\
\hline Frech (38) & $>6$ & ---- & ---- & ---- & ----- & ---- & ---- & $-\cdots$ & ---- \\
\hline Salvadeo (28) & $12 \pm 4$ & ----- & ---- & ---- & ----- & ---- & ---- & ---- & --- \\
\hline \multirow[t]{2}{*}{ Buratti $(39,40)$} & $0.8-11$ & & & & & & & & \\
\hline & $\begin{array}{c}2.4-4.9 \\
7-6.8 \mu \mathrm{g} / \mathrm{L}\end{array}$ & $0.7-13.3 \mu \mathrm{g} / \mathrm{L}$ & ---- & --- & - & ---- & ---- & ---- & \\
\hline Mattiello (29) & $1.5-16.2$ & --. & - & --- & ---- & - & $\cdots$ & --.- & $-\cdots$ \\
\hline Monteagudo (41) & $>10$ & ---- & ---- & ---- & ---- & ---- & --.- & --- & $-\cdots$ \\
\hline Sabbioni (42) & $0.45-8.27$ & $1-29.7 \mu \mathrm{g} / \mathrm{L}$ & ---- & ---- & ---- & ---- & ---- & ---- & $\cdots$ \\
\hline Wang (43) & $>5$ & $>0.5 \mu \mathrm{mol} / \mathrm{L}$ & ---- & ---- & ---- & $-\cdots$ & ---- & --- & --- \\
\hline Kaehny (44) & ----- & $15 \mu \mathrm{g} / \mathrm{die}$ & -.-- & ---- & ---- & ----- & ---- & --- & $-\cdots$ \\
\hline Recker (45) & ---- & $85 \pm 64.9 \mu \mathrm{g} / \mathrm{L}$ & ---- & ---- & ---- & ---- & --- & --- & 0207 \\
\hline Crapper (46) & ---- & ---- & $-\cdots$ & ---- & ---- & ---- & ---- & -- & $0.2-9.7$ \\
\hline McDermott (46) & ---- & ---- & ---- & ---- & $\cdots$ & --- & --- & 1.3 & $2.4-3.9$ \\
\hline Le Gendre (46) & ----- & $-\cdots$ & $-\cdots$ & 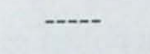 & $-\cdots$ & $-\cdots$ & - & $0 . \overline{7 \pm 0.3}$ & $\begin{array}{c}2.4-3.9 \\
-----\end{array}$ \\
\hline Arieff (46) & ---- & --- & --- & ---- & ---- & --- & ---- & $\begin{array}{l}1.9 \\
1.9\end{array}$ & ----- \\
\hline $\begin{array}{l}\text { Crapper (46) } \\
\text { Alfrey (47) }\end{array}$ & $1.6 \pm 7.5$ & $13-83 \mu \mathrm{g} / \mathrm{die}$ & $2.6 \pm 2.1$ & $4.1 \pm 1.7$ & $1.2 \pm 1.2$ & $1.0 \pm 0.8$ & $43 \pm 44$ & $2.2 \pm 1.3$ & $3.3 \pm 2.9$ \\
\hline $\begin{array}{l}\text { Alfrey (47) } \\
\text { Andersen (21) }\end{array}$ & $\begin{array}{c}1.6 \pm 7.5 \\
---\end{array}$ & $8.3 \pm 1.4 \mu \mathrm{g} / \mathrm{L}$ & -..- & --- & ---- & --.- & ----- & ---- & $0.6 \pm 1.7$ \\
\hline Minoia (22) & $-\cdots$ & --- & --.-- & ----- & ---- & ---- & ---- & ---.- & $1.9-10.62$ \\
\hline
\end{tabular}


TABELLA III - CONCENTRAZIONE DI ALLUMINIO (M \pm DS) NEI TESSUTI E NEI LIQUIDI BIOLOGICI DI PAZIENTI DIALIZZATI VIVENTI E DECEDUTI E NEI SOGGETTI DI CONTROLLO

\begin{tabular}{lccccc}
\hline \multicolumn{2}{c}{ Dializzati } & \multicolumn{2}{c}{ Controlli } & P \\
\hline & $\mathrm{M} \pm \mathrm{DS}$ & Soggetti & $\mathrm{M} \pm \mathrm{DS}$ & Soggetti & \\
\hline Urina (pazienti viventi) & $39.3 \pm 67.2$ & 47 & $4.1 \pm 4.3$ & 45 & 0.00005 \\
Siero (pazienti viventi) & $42.9 \pm 29.2$ & 111 & $19 \pm 7.5$ & 45 & 0.00005 \\
Urina (casi autoptici) & $81 \pm 79$ & 4 & $20 \pm 7$ & 4 & n.s. \\
Sangue (casi autoptici) & $161 \pm 202$ & 10 & $9 \pm 4$ & 10 & 0.0002 \\
Bile & $185 \pm 191$ & 9 & $5 \pm 2$ & 10 & 0.0002 \\
Cervello & $5 \pm 3$ & 9 & $1 \pm 1$ & 10 & 0.0008 \\
Costa & $8 \pm 9$ & 11 & $1 \pm 1$ & 10 & 0.0022 \\
Cartilagine & $9 \pm 9$ & 10 & $1 \pm 1$ & 10 & 0.0002 \\
Cranio & $11 \pm 12$ & 7 & $1 \pm 1$ & 10 & 0.0012 \\
Polmone & $55 \pm 46$ & 12 & $12 \pm 12$ & 10 & 0.0016 \\
Cuore & $6 \pm 6$ & 11 & $2 \pm 1$ & 7 & n.s. \\
Milza & $17 \pm 13$ & 12 & $8 \pm 13$ & 10 & 0.0241 \\
Rene & $7 \pm 7$ & 12 & $2 \pm 1$ & 10 & 0.0001 \\
Aorta & $8 \pm 10$ & 11 & $1 \pm 1$ & 8 & 0.0001 \\
Vena Cava & $5 \pm 5$ & 11 & $1 \pm 1$ & 10 & 0.0029 \\
Fegato & $17 \pm 20$ & 12 & $2 \pm 1$ & 10 & 0.0009 \\
Muscolo & $4 \pm 3$ & 12 & $1 \pm 1$ & & 0.0100
\end{tabular}

$+0.1 \%$ di Triton X-100, Merk, in acqua deionizzata e bidistillata) e, dopo vortex per 1 minuto, i campioni, trasferiti negli appositi vials conici (Varian), sono stati posti nell'autocampionatore. Mediante probe automatico $10 \mu \mathrm{l}$ sono stati depositati nel tubo pirolitico ed analizzati. La curva di calibrazione è stata costruita utilizzando un pool di siero o di urina a bassa concentrazione su cui sono state operate aggiunte di soluzioni di alluminio a concentrazione nota ottenute mediante diluizione della soluzione standard di Alluminio Spectrosol al titolo di $1 \mathrm{~g} / \mathrm{L}(\mathrm{BDH})$. Infine sono stati esaminati campioni di siero, forniti dall'Istituto Superiore di Sanità, contenenti quantità note di alluminio al fine di verificare, per ogni determinazione, l'attendibilità del risultato.

L'analisi statistica dei dati ottenuti è stata condotta con metodi non parametrici utilizzando il Test di Wilcoxon.

\section{Risultati}

\section{Determinazioni seriche ed urinarie nei soggetti viventi}

Nessuna differenza significativa per quanto riguarda età e distribuzione secondo il sesso è stata rilevata tra soggetti in emodialisi e gruppo di controllo.

I sieri dei soggetti in emodialisi hanno mostrato valori di alluminemia di $42.9 \pm$ $29.2 \mu \mathrm{g} / \mathrm{L}$, mentre i soggetti di controllo hanno mostrato concentrazioni di alluminio ematico di $19.0 \pm 7.5 \mu \mathrm{g} / \mathrm{L}$, con una differenza altamente significativa $(\mathrm{p}=0.00005)$ (Tab. III).

Le urine dei soggetti dializzati hanno mostrato valori di alluminio di $39.3 \pm$ $67.2 \mu \mathrm{g} / \mathrm{L}$, mentre quelle dei soggetti normali hanno mostrato valori di $4.1 \pm$ $4.3 \mu \mathrm{g} / \mathrm{L}$, con una differenza altamente significativa $(\mathrm{p}=0.00005)$.

È stata riscontrata una correlazione positiva e significativa tra livelli ematici e livelli urinari di alluminio nella globalità della popolazione esaminata $\quad(r=0.503$; $\mathrm{p}=0.00005$ ).

Nella casistica considerata, i livelli ematici ed urinari di alluminio non sono risultati correlati con l'età.

\section{Determinazioni tessutali, seriche ed urinarie in soggetti deceduti}

Tra il gruppo dei soggetti dialitici sottoposti ad autopsia ed il gruppo di controllo sottoposto ad autopsia, non è stata rilevata alcuna differenza significativa per quanto riguarda l'età $(70.25 \pm 11.10$ vs $56.40 \pm 23.94$ anni) e la distribuzione re- lativa al sesso.

Le concentrazioni tessutali di alluminio nei soggetti dialitici e di controllo sono esposte nella Tabella III. In tutti i tessuti esaminati i livelli di alluminio sono risultati superiori nei dializzati rispetto ai controlli: la differenza è statisticamente significativa in tutti i tessuti eccetto che nel cuore e nelle urine; bisogna rilevare che durante il riscontro autoptico è stato possibile raccogliere campioni di urine solo da quattro soggetti in emodialisi e da quattro controlli.

Abbiamo poi esaminato separatamente le concentrazioni tessutali di alluminio nei dializzati e nei controlli, confrontando fra loro i livelli nei vari tessuti: le differenze significative sono esposte nelle $\mathrm{Ta}$ belle IV e V, rispettivamente per i soggetti dialitici e per i controlli.

I valori più elevati riscontrati nei soggetti dializzati sono stati quelli a livello della bile, seguiti dal sangue, dalle urine e dal polmone; tutti gli altri tessuti seguono a distanze considerevoli.

Nei controlli questa distribuzione è solo parzialmente sovrapponibile, essendo decisamente basso l'accumulo nel fegato e nella bile, a differenza dei dializzati. Si confermano invece valori sorprendentemente elevati e mai prima descritti nel polmone sia dei dializzati che dei controlli: in questi ultimi i livelli polmonari 


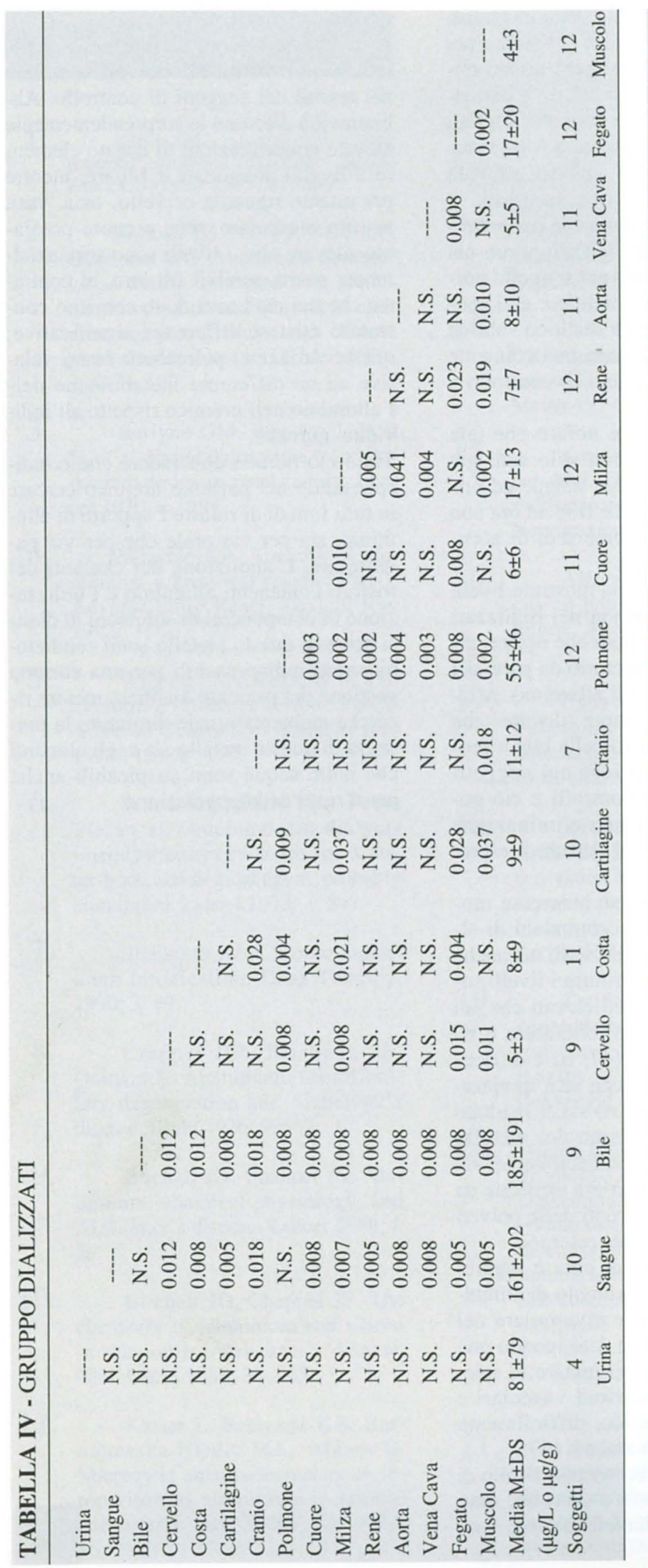

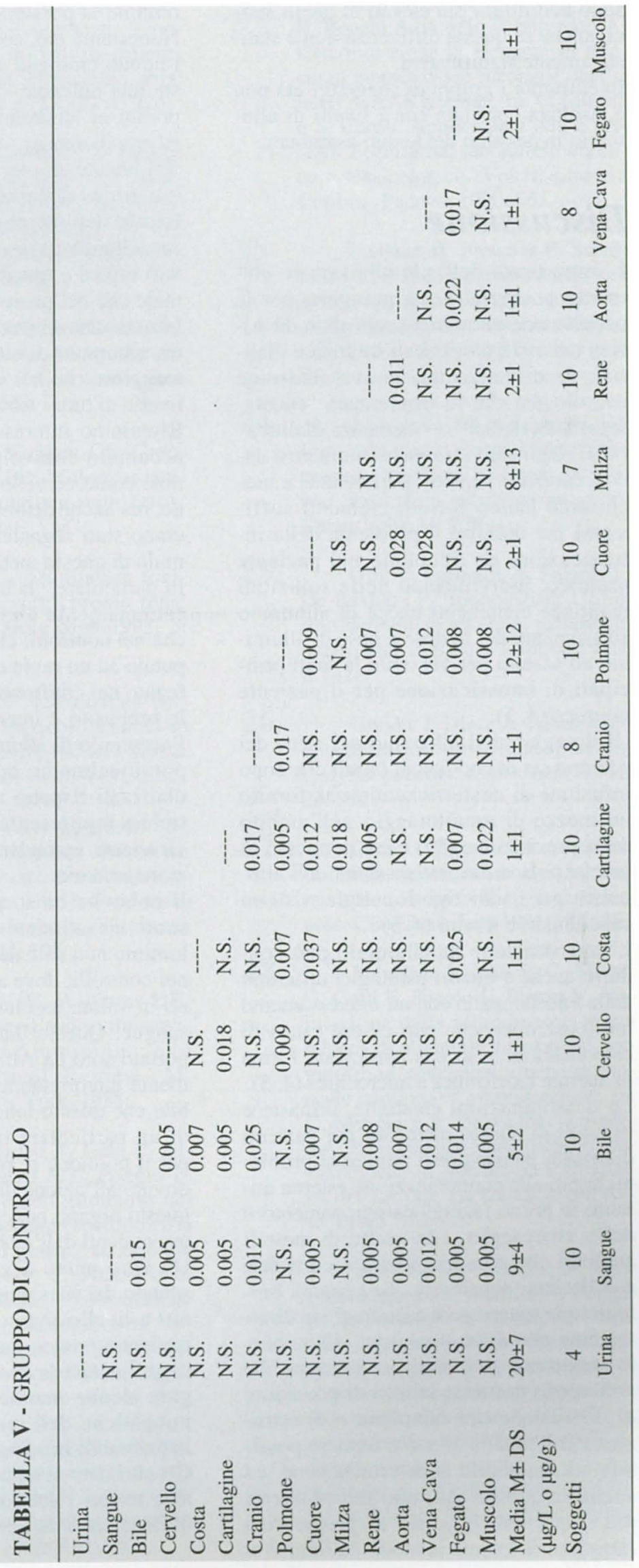


sono addirittura più elevati di quelli serici, anche se questa differenza non è statisticamente significativa.

In entrambi i gruppi di soggetti l'età non è risultata correlata con i livelli di alluminio in nessuno dei tessuti esaminati.

\section{Discussione}

L'importanza dell'alluminio quale elemento potenzialmente patogeno per la popolazione uremica fu segnalato da $\mathrm{Al}$ frey nel 1972 su pazienti uremici e dialitici, descrivendo una nuova sindrome neurologica che fu denominata "encefalopatia da dialisi" o " demenza dialitica" (48). Negli anni successivi, numerosi dati di carattere clinico, sperimentale e biochimico hanno fornito elementi sufficienti per definire il problema della intossicazione da alluminio nel paziente uremico, individuando nelle soluzioni dialitiche contenenti tracce di alluminio ed i chelanti del fosforo a base di alluminio ed assunti per via orale le fonti principali di intossicazione per il paziente uremico $(4,5)$.

Il dosaggio dell'alluminio nel siero dei pazienti sia in condizioni basali che dopo infusione di desferrioxamina ha fornito un mezzo di monitoraggio nell'ambito della prevenzione (17) e ha permesso di precisare la differente tossicità dell'alluminio per i vari tipi di cellule, sistemi subcellulari e tessuti $(4,5)$.

L'intossicazione da alluminio può condurre anche a quadri patologici differenti dalla encefalopatia con un interessamento multisistemico che può essere causa di osteomalacia e di una particolare forma di anemia ipocromica e microcitica $(4,5)$. Le determinazioni ematiche, urinarie e tissutali di questo elemento nei pazienti dializzati hanno posto numerosi problemi legati alla contaminazione esterna durante le prime fasi del campionamento o dello stoccaggio, alla scelta di metodi analitici che possono offrire accuratezza e sufficiente sensibilità. Se i liquidi biologici possono essere esaminati sia direttamente che dopo opportuna diluizione, lo stesso non può dirsi per i tessuti che richiedono la messa in atto di procedure di distruzione del campione o di estrazione dell'analita adatte a rendere possibile ed attendibile la determinazione, ed anche in questo caso uno dei problemi più importanti è quello di preparare $\mathrm{i}$ campioni di tessuto in modo da ridurre al minimo la possibilità di contaminazione. Nonostante ciò, così come si verifica per i liquidi biologici, anche per i tessuti esiste una notevole variabilità tra $i$ dati riportati in letteratura in soggetti normali ed emodializzati, variabilità dovuta verosimilmente alla tecnica analitica adottata e ai gruppi di popolazione considerati.

I nostri dati rappresentano una panoramica della distribuzione dell'alluminio nei vari organi e tessuti sia nel soggetto normale che nel paziente dialitico: essi confermano che il paziente dialitico subisce un accumulo di alluminio notevolmente maggiore che nei soggetti di controllo a livello di tutti i tessuti.

Riteniamo interessante notare che tale accumulo coinvolge non solo sedi già note come cervello, ossa, sangue ed urine, ma anche distretti che fino ad ora non erano stati segnalati come sedi di accumulo di questo metallo.

In particolare, la bile ha mostrato livelli estremamente elevati sia nei dializzati che nei controlli; ciò potrebbe essere imputato ad un ruolo escretorio da parte del fegato nei confronti dell'alluminio. A tale proposito è interessante rilevare che l'accumulo di alluminio nella bile è proporzionalmente maggiore nei soggetti dializzati rispetto ai controlli e ciò potrebbe rappresentare una eliminazione vicariante secondaria al deficit di escrezione urinaria.

Il polmone ha sorprendentemente mostrato elevatissime concentrazioni di alluminio non solo nei dializzati, ma anche nei controlli, dove addirittura i livelli sono in valore assoluto più elevati che nel sangue. Questo dato in letteratura è riportato solo da Alfrey (47) ed è difficilmente interpretabile anche se è ipotizzabile che questo fenomeno sia il risultato di un particolare tropismo del metallo per il polmone $o$, più probabilmente, sia dovuto all'azione di barriera esplicata da questo organo nei confronti delle polveri provenienti dall' ambiente esterno.

Un altro punto degno di nota è rappresentato dal rilevante accumulo di alluminio a livello muscolare e vascolare nei pazienti uremici; questo dato inedito può rappresentare la base per tentare di spiegare alcune manifestazioni vascolari e miopatiche dell'uremico, difficilmente inquadrabili nosologicamente (49).

Gli altri dati da noi riscontrati a livello di altri tessuti, quali ossa e cervello, sono in linea con $\mathrm{i}$ dati della letteratura e sono già stati ampiamente discussi.

Altre considerazioni da fare sono quelle relative alla distribuzione dell'alluminio nei tessuti dei soggetti di controllo. Abbiamo già discusso le sorprendentemente elevate concentrazioni di questo elemento a livello polmonare e biliare, mentre per quanto riguarda cervello, ossa, vasi, tessuto muscolare, rene e cuore possiamo rilevare che i livelli sono sostanzialmente sovrapponibili fra loro, al contrario che nei dializzati dove abbiamo constatato esistere differenze significative; queste variazioni potrebbero essere relative ad un differente metabolismo dell'alluminio nell'uremico rispetto all'individuo normale.

Tutto ciò porta a concludere che è indispensabile nel paziente uremico cercare in tutti i modi di ridurre l'apporto di alluminio, sia per via orale che per via parenterale. L'abolizione dei chelanti del fosforo contenenti alluminio e l'utilizzazione di componenti di soluzioni di dialisi prive di questo metallo sono condizioni ormai indispensabili per una corretta gestione del paziente dialitico, mentre ricerche ambientali onde diminuire la presenza di questo metallo sia negli alimenti che nelle acque sono auspicabili anche per il resto della popolazione. 
BIBLIOGRAFIA

1. Klein GL. Aluminium in parenteral solutions revisited - again. Am J Clin Nutr 1995; 61: 449.

2. Salusky IB, Foley J, Nelson P, Goodman WG. Aluminium accumulation during treatment with aluminium hydroxide and dialysis in children and young adults with chronic renal disease. New Eng J Med 1991; 324 (8): 527.

3. Berlyne GM, Ben-Ari J, Pest D, et al. Hyperaluminemia from aluminium resins in renal failure. Lancet 1970; i: 494.

4. Winship KA. Toxicity of aluminium: a historical review. Part 1 . Adverse Drug React. Toxicol Rev $1992 ; 11(2): 123$.

5. Winship KA. Toxicity of aluminium: a historical review. Part 2. Adverse Drug React Toxicol Rev $1993 ; 12(3): 177$.

6. Ward MK, Ellis HA, Feest TG, et al. Osteomalacic dialysis osteodystrophy: evidence for a water-bone aetiological agent, probably aluminium. Lancet 1978; i: 841.

7. Shalhoub RJ. Chronic aluminium intoxication. Drug Therapy, 1990; 3: 99.

8. Crapper DR, Krishnan SS, Quittkat S. Aluminium, neurofibrillary degeneration and Alzheimer's disease. Brain 1976; 99: 67.

9. Birchall JD, Chappel JS. Aluminium, chemical physiology, and Alzheimer's disease. Lancet 1988; i: 59.

10. Birchall JD, Chappel JS. The chemistry of aluminium and silicon in relation to Alzheimer's disease. Clin Chem 1988; 34: 265.

11. Kaiser L, Schwartz KA, Burnotowska-Hledin MA, Mayor G. Microcytic anemia secondary to intoperitoneal aluminium in normal and uremic rats. Kidney Int 1984; 26: 269.
12. Short AIK, Winney RJ, Robson JS. Reversible microcytic hypochromic anemia in dialysis patients due to aluminium intoxication. Proc Europ Dial Trans Ass 1980; 7: 226.

13. Tovan M, Martinez F, Lacour B, Bourdon R, Zingraff J. Aluminium-induced reversible microcytic anemia in chronic renal failure: clinical and experimental studies. Clin Nephrol 1983; 19: 95.

14. Cann CE, Prussin SG, Gordan GS. Aluminium uptake by the parathyroid glands. J Clin Endocrinol Metab 1979; 9: 543.

15. Karlik SJ, Eichorn GL, Crapper McLachaln DR. Molecular interactions of aluminium with DNA. Neurotoxicol 1986; 5: 83.

16. Alfrey AC. Aluminium toxicity in patients with chronic renal failure. Therap Drug Mon 1993; 5: 93.

17. Canavese C. La tossicità dell'alluminio nei pazienti uremici in trattamento dialitico. Italian J Min \& Electrolite Metab, 1990; 4 (3): 201.

18. Reusche E, Lindner B, Arnholdt H. Widespread aluminium deposition in extracerebral organ systems of patients with dialysis-associated encephalopathy. Virchows Archiv 1994; 424: 105.

19. Duffield VJR, Williams DR. Aluminium in food and in the enviroment. In: Aluminium and Health.A Critical Review. M. Dekker Ed., New York, 1988.

20. Minoia C, Salvadeo A, Galli P, Aldrighetti G, Vitali MT, Poggio F. Determinazione dell'alluminio nel tessuto osseo mediante spettrometria di emissione al plasma accoppiato induttivamente. In: C. Minoia e A. Salvadeo. Alluminio. Problematiche analitiche e Tossicologiche in Nefrologia e Medicina del Lavoro. La Goliardica Pavese, Pavia, 1984; 203.

21. Andersen JR, Reimert S. Determination of aluminium in human tissues and body fluids by Zeemancorrected atomic absorption spectrometry. Analyst 1986; 111: 657.
22. Minoia C, Sabbioni E, Pietra R, Ronchi A, Poggio F, Salvadeo A. Determinazione di elementi in traccia in biopsie ossee mediante analisi in ETA-AAS Zeeman. In: C. Minoia e S. Caroli. Applicazioni dell'ETAAAS Zeeman nel laboratorio chimico e tossicologico. Vol II, Libreria Cortina, Padova, 1990; 661.

23. Valentin H, Preusser P, Schaller K. Die Analyse von Aluminium in Serum und Urin zur veberwachung exponierter Personer. Int Archiv Occup Environ Health 1976; 38: 1.

24. Alderman FR, Gitelman HJ Improved electrothermal determination of aluminium in serum by atomic absorption spectroscopy. Clin Chem 1980; 26: 258.

25. Gilli P, Farinelli A, Fagioli F De Bastiani P, Buoncristiani U. Serum aluminium levels in patients in peritoneal dialysis. Lancet 1980 ; ii:742.

26. Gorsky J, Dietz A. Aluminium concentrations in serum of hemodialysis patients. Clin Chem 1981; 27: 932 .

27. Oster $\mathrm{O}$. The aluminium content of human serum determined by atomic spectroscopy with a graphite furnace. Clin Chem Acta 1981; 114: 53.

28. Salvadeo A, Minoia C, Micoli G, Galli F, Poggio F, Piazza V. Valutazione della alluminiemia e dei bilanci di alluminio in pazienti sottoposti ad emodialisi, dialisi peritoneale continua ed emofiltrazione. In: C. Minoia e A. Salvadeo. Alluminio. Problematiche analitiche e tossicologiche in Nefrologia e Medicina del Lavoro. La Goliardica Pavese, Pavia, 1984; 51.

29. Mattiello G, Salvadeo A, Galli P, Aldrighetti G, Vitali MT, Poggio F. Determinazione diretta dell'alluminio nel siero mediante spettroscopia di assorbimento atomico con atomizzazione elettrotermica e modificazione di matrice. In: C. Minoia e A. Salvadeo Alluminio. Problematiche analitiche e tossicologiche in Nefrologia e Medicina del Lavoro. La Goliardica Pavese, Pavia, 1984; 151. 
30. Sanna T, Conti M, Cadeddu $\mathrm{M}$, et al. Il problema dell'alluminio in Sardegna: studio sugli emodializzati della Provincia di Cagliari. In: Atti del Congresso La tossicità dell'Alluminio. A cura di S. Costantini e R. Giordano, Roma, 21 settembre 1989.

31. Cecco A, Menin L, Tommasi J, Peretti A. Determinazione di alluminio: controlli di qualità ed indagini applicative. In: Atti del Congresso. La tossicità dell'Alluminio. A cura di S. Costantini e R. Giordano, Roma 21 settembre 1988.

32. Aucella F, Guida C, Napolitano G, Di Giorgi G, Stallone C. Alluminiemia:screening in un centro di dialisi. In: Atti del Congresso La tossicità dell'alluminio. A cura di S. Costantini e R. Giordano, Roma 21 settembre 1988 .

33. Copercini B, Bolzoni G, Margonari M, Pecchini F, Somenzi V. Intossicazione da alluminio: risultati preliminari di una indagine negli emodializzati del centro di Cremona. In Atti del Congresso. La tossicità dell'Alluminio. A cura di S. Costantini e R. Giordano, Roma, 21 settembre 1989 .

34. Clavel J, Jaudon M, Galli A. Dosage de l'aluminium dans les liquides biologiques par spectrophotométrie atomique en forn graphite. Annals de Biologie Clinique, 1978; 36: 33 .

35. Allain AJ, Mauras Y. Determination of aluminium in blood, urine and water by inductively coupled plasma emission spectometry. Analytical Chemistry 1979; 51: 2089.

36. Leung F, Henderson A. Improved determination of aluminium in serum and urine with use of a stabilized temperature platform furnace. Clin Chem 1982; 28: 2139.

37. Parkinson I, Ward M, Kerr D. A method for the routine determination of aluminium in serum and water by flameless atomic absorption spectrometry. Clin Chem Acta 1982; 125: 125.

38.

Frech W, Cedergreen A, Ce- derberg C, Vessman J. Evaluation of some critical factors affecting determination of aluminium in blood, plasma or serum by electrothermal atomic absorption spectroscopy. Clin Chem 1982; 28: 2259.

39. Buratti M, Caravelli G, Calzaferri G, Colombi A. Determination of aluminium in body fluids by solvent extraction and atomic absorption spectroscopy with electrothermal atomization. Clinical and Chimical Acta 1984; 141: 253.

40. Buratti M, Caravelli G, Calzaferri G, Colombi A. Determinazione dell'Alluminio nei liquidi biologici mediante un metodo estrattivo e spettrometria di assorbimento atomico con atomizzazione elettrotermica. In: C. Minoia e A. Salvadeo. Alluminio. Problematiche analitiche e tossicologiche in Nefrologia e Medicina del Lavoro. La Goliardica Pavese, Pavia, 1984; 163.

41. Monteagudo FSE, Sassidy MJD, Folb PI. Recent developments in aluminium toxicology. Medical Toxicology 1989; 4: 1 .

42. Sabbioni E, Apostoli P, Minoia C. Impiego dell'ETA-AAS Zeeman nella definizione dei valori di riferimento di 22 elementi in traccia nei liquidi biologici. In: C. Minoia e S. Caroli Applicazioni dell'ETA-AAS Zeeman nel laboratorio chimico e tossicologico. Vol. II, Libreria Cortina, Padova, 1990; 371.

43. Wang ST, Pizzolato S, Demshar HP. Aluminium levels in normal human serum and urine as determined by Zeeman Atomic Absorption Spectrometry. Journal of Analytical Toxicology 1991; 15: 66.

44. Kaehny WD, Hegg AP, Alfrey AC. Gastrointestinal absorption of aluminium from aluminium-containing antiacids. New Engl J Med 1977; 296: 1389.

45. Recker RR, Blotcky AJ, Leffler JA, Rack EP. Evidence for aluminium absorption from the gastrointestinal tract and bone deposition by aluminium carbonate ingestion with normal renal function. Journal of Laboratory and Clinical
Medicine, 1977; 90: 810.

46. Cedergren A, Ferewck W. Determination of aluminium in biological materials by graphite furnace atomic absorption spectrometry (GFAAS). Pure Applied Chemistry 1987; 59: 222.

47. Alfrey AC. Physiology of aluminium in man. In: Aluminium and Health. M. Dekker Ed., New York, 1989.

48. Alfrey AC, Mischell JM, Brurks J. Syndrome of dyspraxia and multifocal seizures associated with chronic hemodialysis. Transplant American Society Artif Intern Organs, 1972; 18: 257.

49. Pierides AM, Ewards WG, Cullum UX, McCall JT, Ellis HA. Haemodialysis encephalopathy with osteomalacic fractures and muscle weakness. Kidney Int 1980; 18: 115. 